\title{
The Profile of Inelastic Collisions from Elastic Scattering Data
}

\author{
I. M. Dremin ${ }^{1,2}$ \\ ${ }^{1}$ Lebedev Physical Institute, Moscow 119991, Russia \\ ${ }^{2}$ National Research Nuclear University "MEPhI", Moscow 115409, Russia \\ Correspondence should be addressed to I. M. Dremin; dremin@lpi.ru
}

Received 15 October 2014; Accepted 14 December 2014

Academic Editor: Edward Sarkisyan-Grinbaum

Copyright (C) 2015 I. M. Dremin. This is an open access article distributed under the Creative Commons Attribution License, which permits unrestricted use, distribution, and reproduction in any medium, provided the original work is properly cited. The publication of this article was funded by $\mathrm{SCOAP}^{3}$.

\begin{abstract}
Using the unitarity relation in combination with experimental data about the elastic scattering in the diffraction cone, it is shown how the shape and the darkness of the inelastic interaction region of colliding protons change with increase of their energies. In particular, the collisions become fully absorptive at small impact parameters at LHC energies that results in some special features of inelastic processes. Possible evolution of this shape with the dark core at the LHC to the fully transparent one at higher energies is discussed that implies that the terminology of the black disk would be replaced by the black toroid. The approach to asymptotics is disputed. The ratio of the real to imaginary parts of the nonforward elastic scattering amplitude is briefly discussed. All the conclusions are only obtained in the framework of the indubitable unitarity condition using experimental data about the elastic scattering of protons in the diffraction cone without any reference to quantum chromodynamics (QCD) or phenomenological approaches.
\end{abstract}

\section{Introduction}

In this paper, our knowledge of the shape and opacity of the interaction region of two colliding protons and the behavior of their elastic scattering amplitude at various transferred momenta at high energies are discussed.

The general common approach to both problems considering the irrefutable statement that the total probability of all possible processes must be equal to 1 will be used. It is called the unitarity condition and will be applied to information stemming from experiment about the elastic scattering of protons at small angles within the diffraction cone for the first problem and at larger angles outside it for the second problem. The generality of the approach guarantees the certainty of the obtained results. At the same time, surely, it cannot substitute the knowledge of the dynamics of the process but helps get some interesting conclusions about the problems to be approached. That is especially important in view of the limited applications of QCD to quantitative description of experimental data. In addition, some results of the phenomenological models are briefly discussed and confronted to our conclusions as well. The usage in the present paper only these two indubitable ingredients-the unitarity condition and experimental results about the elastic scattering-is decisive for the confidence in derived conclusions.

\section{The General Approach and Results}

Colliding high energy hadrons can either scatter elastically when only two of them appear at the final stage without changing their nature or produce some new particles in inelastic processes. Kinematics of elastic scattering is very simple. It is described by two variables: the squared total energy $s=4 E^{2}$, where $E$ is the energy of one of partners in the center of mass system, and the four-momentum transfer squared $-t=2 p^{2}(1-\cos \theta)$ with $\theta$ denoting the scattering angle and $p$ the momentum in the center of mass system. For inelastic processes the kinematics is much more complicated. Therefore, to avoid some complications, it is quite natural to try to get at the first stage some knowledge about the dynamics of the whole process starting from the analysis of elastic scattering and using such general relation as the unitarity condition. It follows from the irrefutable statement that the total probability of all (elastic + inelastic) processes 
should be equal to 1 . In this way it relates these two channels of the reaction albeit in rather average integrated form. It is the mainstream of the approach adopted in the present paper.

The only information about elastic scattering at a fixed energy $s$ comes from the measurement of the differential cross section as a function of the transferred momentum $t$ at its experimentally available values and of the ratio of the real and imaginary parts $\rho(s, t)=\operatorname{Re} f(s, t) / \operatorname{Im} f(s, t)$ of the elastic scattering amplitude $f(s, t)$ just in forward direction $t=0 \rho(s, 0)=\rho_{0}$ but not at any other values of $t$. The latter one is obtained from studies of interference between the nuclear and Coulomb contributions to the amplitude $f$ which becomes practically noticeable only in the nearforward direction.

The differential cross section is related to the scattering amplitude as

$$
\frac{d \sigma}{d t}=|f(s, t)|^{2}
$$

Thus, from measurements of the differential cross section at any energy of colliding particles, we get the knowledge only about the modulus of the amplitude at the experimentally available values of $t$. As a first approximation at the present energies, it can be described at comparatively small transferred momenta by the exponential shape with the slope $B$ such that

$$
\frac{d \sigma}{d t}=\frac{\sigma_{t}^{2}}{16 \pi} \exp (-B|t|) .
$$

This region is called the diffraction peak. It becomes higher and its width shrinks with increasing energy because both the total cross section $\sigma_{t}$ and the slope $B$ increase with energy.

The unitarity of the $S$-matrix $S S^{+}=1$ imposes definite requirements on the amplitude of elastic scattering $f(s, t)$ and amplitudes of inelastic processes $M_{i}$. In the $s$-channel it looks [1-3] like

$$
\begin{aligned}
\operatorname{Im} & f(p, \theta) \\
= & I_{2}(p, \theta)+g(p, \theta) \\
= & \frac{s}{8 \pi^{3 / 2}} \iint d \theta_{1} d \theta_{2} \\
& \cdot \frac{\sin \theta_{1} \sin \theta_{2} f\left(p, \theta_{1}\right) f^{*}\left(p, \theta_{2}\right)}{\sqrt{\left[\cos \theta-\cos \left(\theta_{1}+\theta_{2}\right)\right]\left[\cos \left(\theta_{1}-\theta_{2}\right)-\cos \theta\right]}} \\
& +g(p, \theta) .
\end{aligned}
$$

The region of integration in (3) is given by the conditions

$$
\left|\theta_{1}-\theta_{2}\right| \leq \theta, \quad \theta \leq \theta_{1}+\theta_{2} \leq 2 \pi-\theta .
$$

The nonlinear integral term represents the two-particle intermediate states of the incoming particles. The function

$$
g(p, \theta) \propto \sum_{i} \int d \Phi_{i} M_{i} M_{i}^{*}(\theta)
$$

represents the shadowing contribution of the inelastic processes to the imaginary part of the elastic scattering amplitude. Following [4] it is called the overlap function. This terminology is ascribed to it because the integral there defines the overlap within the corresponding phase space $d \Phi_{i}$ between the matrix element $M_{i}$ of the ith inelastic channel and its conjugated counterpart with the collision axis of initial particles turned by the angle $\theta$ of proton scattering in the elastic process. It is positive at $\theta=0$ but can change sign at $\theta \neq 0$ due to the relative phases of inelastic matrix elements $M_{i}$ 's.

At $t=0$ it leads to the optical theorem

$$
\operatorname{Im} f(s, 0)=\frac{\sigma_{t}}{4 \sqrt{\pi}}
$$

and to the general statement that the total cross section is the sum of cross sections of elastic and inelastic processes:

$$
\sigma_{t}=\sigma_{\mathrm{el}}+\sigma_{\mathrm{in}}
$$

that is, the total probability of all processes is equal to one.

That allows to estimate the real and imaginary parts separately just in forward direction $t=0$ after the values of $\rho_{0}$ and $\sigma_{t}$ are measured.

To define the geometry of the collision we must express all characteristics presented by the angle $\theta$ and the transferred momentum $t$ in terms of the transverse distance between the centers of the colliding protons called the impact parameter $b$. It is easily done by the Fourier-Bessel transform of the amplitude $f$ which retranslates the momentum data to the transverse space features and is written as

$$
i \Gamma(s, b)=\frac{1}{2 \sqrt{\pi}} \int_{0}^{\infty} d|t| f(s, t) J_{0}(b \sqrt{|t|}) .
$$

The unitarity condition in the $b$-representation reads

$$
G(s, b)=2 \operatorname{Re} \Gamma(s, b)-|\Gamma(s, b)|^{2} .
$$

The left-hand side (the overlap function in $b$-representation) describes the transverse impact-parameter profile of inelastic collisions of protons. It is just the Fourier-Bessel transform of the overlap function $g$. It satisfies the inequalities $0 \leq$ $G(s, b) \leq 1$ and determines how absorptive the interaction region is depending on the impact parameter (with $G=$ 1 for the full absorption and $G=0$ for the complete transparency). The profile of elastic processes is determined by the subtrahend in (9).

Let us note the general structure of the above expression. Defining $\operatorname{Re} \Gamma(s, b)=2 v$ and $\operatorname{Im} \Gamma(s, b)=2 \rho v$, one gets

$$
G(s, b)=4\left[\nu(1-v)-\rho^{2} v^{2}\right] .
$$

It is clearly seen therefrom that $G(s, b) \leq 1$, and $G(s, b)=1$ only if $\nu=0.5, \rho=0$. It is less than 1 at any other arbitrary values of $\nu$ and $\rho$.

Even though the impact parameter cannot be directly measured, the geometric picture is instructive and closely related to such experimentally found characteristics as the 


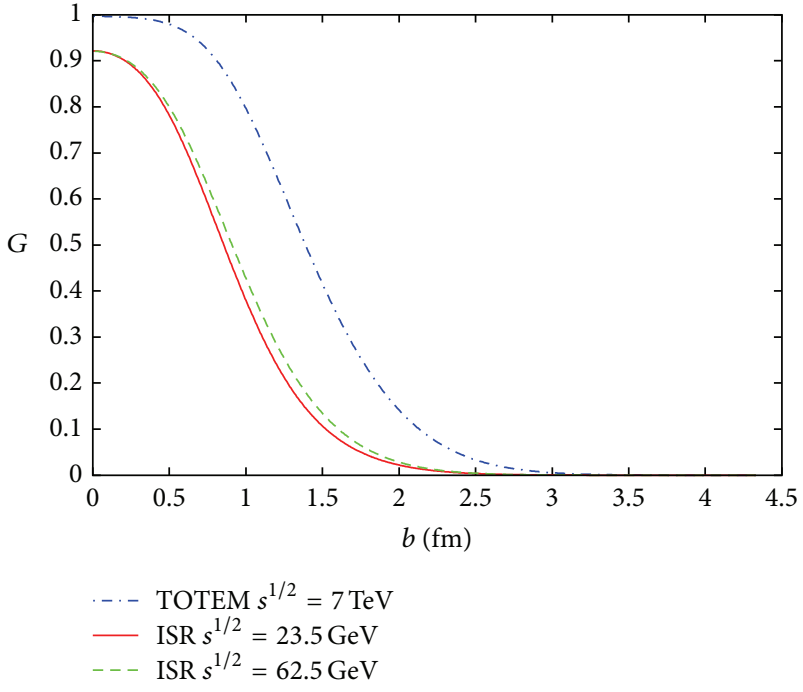

(a)

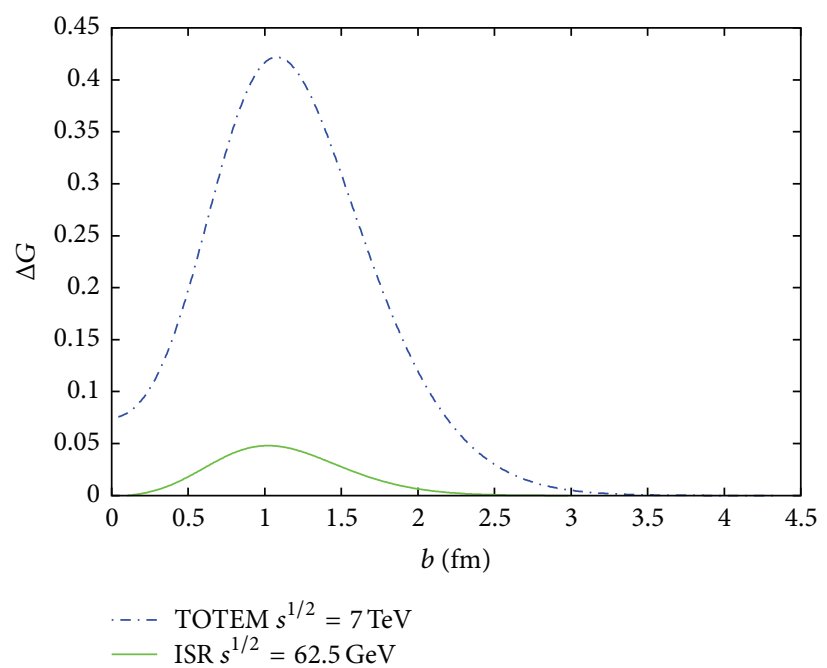

(b)

Figure 1: (a) The overlap function $G(s, b)$ at $7 \mathrm{TeV}$ (upper curve) [5] compared to those at ISR energies $23.5 \mathrm{GeV}$ and $62.5 \mathrm{GeV}$. (b) Their differences (computed using the fit of experimental data by the model [6]).

ratio of the diffraction cone slope to the total cross section that provides immediate guides to its energy evolution. The impact parameter profiles of elastic and inelastic hadron collisions are derived as Fourier-Bessel transforms of the measurable data. They help us visualize the geometrical picture of partonic interactions indicating their space extension and the intensity. Our intuitive guesses about the space-time development of these processes can be corrected in this way.

The diffraction cone contributes mostly to the FourierBessel transform of the amplitude. Using the above formulae, one can write the dimensionless $\Gamma$ as

$$
\begin{aligned}
& i \Gamma(s, b) \\
& \quad=\frac{\sigma_{t}}{8 \pi} \int_{0}^{\infty} d|t| \exp \left(-\frac{B|t|}{2}\right)(i+\rho(s, t)) J_{0}(b \sqrt{|t|}) .
\end{aligned}
$$

Here, the diffraction cone approximation (2) is inserted. Then one calculates

$$
\operatorname{Re} \Gamma(s, b)=\frac{1}{Z} \exp \left(-\frac{b^{2}}{2 B}\right)
$$

where we introduce the dimensionless ratio of the cone slope (or $\sigma_{\mathrm{el}}$ ) to the total cross section:

$$
Z=\frac{4 \pi B}{\sigma_{t}}=\frac{\sigma_{t}}{4 \sigma_{\mathrm{el}}} .
$$

As was mentioned, the ratio $\rho(s, t)$ is very small at $t=0$ and, at the beginning, we neglect it and get very robust formula

$$
G(s, b)=\frac{2}{Z} \exp \left(-\frac{b^{2}}{2 B}\right)-\frac{1}{Z^{2}} \exp \left(-\frac{b^{2}}{B}\right) .
$$

For central collisions with $b=0$ it gives

$$
G(s, b=0)=\frac{2 Z-1}{Z^{2}} .
$$

TABle 1: The energy behavior of $Z$ and $G(s, 0)$ obtained from experiment.

\begin{tabular}{lccccccccc}
\hline$\sqrt{s}, \mathrm{GeV}$ & 2.70 & 4.11 & 4.74 & 7.62 & 13.8 & 62.5 & 546 & 1800 & 7000 \\
\hline$Z$ & 0.64 & 1.02 & 1.09 & 1.34 & 1.45 & 1.50 & 1.20 & 1.08 & 1.00 \\
$G(s, 0)$ & 0.68 & 1.00 & 0.993 & 0.94 & 0.904 & 0.89 & 0.97 & 0.995 & 1.00
\end{tabular}

Thus, the darkness at the very center is fully determined by the parameter $Z$, that is, by the ratios of experimentally measured characteristics - the width of the diffraction cone $B$ (or the elastic cross section) to the total cross section. Their energy evolution defines the evolution of the absorption value. The interaction region becomes completely absorptive in the center only at $Z=1$ and the absorption diminishes for other values of $Z$. At ISR energies $Z \approx 1.5$; then it decreases systematically and at LHC energies becomes equal to 1 within the accuracy of measurements of $B$ and $\sigma_{t}$ (see Table 1) which can be roughly estimated at the level about $10^{-2}$. The general analysis of the cone behavior at different energies is awaited for.

We stress that all above conclusions are robust for the single-exponent fit of the differential cross section (2). More complicated fits should be studied separately.

The energy evolution of the inelastic profile $G(s, b)$ is shown in Figure 1.

The central core is very absorptive at LHC. It is responsible for jets [7]. It is possible [8] to use this information from elastic processes for analysis of experimental data of CMS collaboration at $7 \mathrm{TeV}$ about high multiplicity inelastic collisions triggered by the hadron jet production [9]. The black plateau in the central part of the interaction region with $b<0.4-0.5 \mathrm{fm}$ should result in the corresponding plateau of 


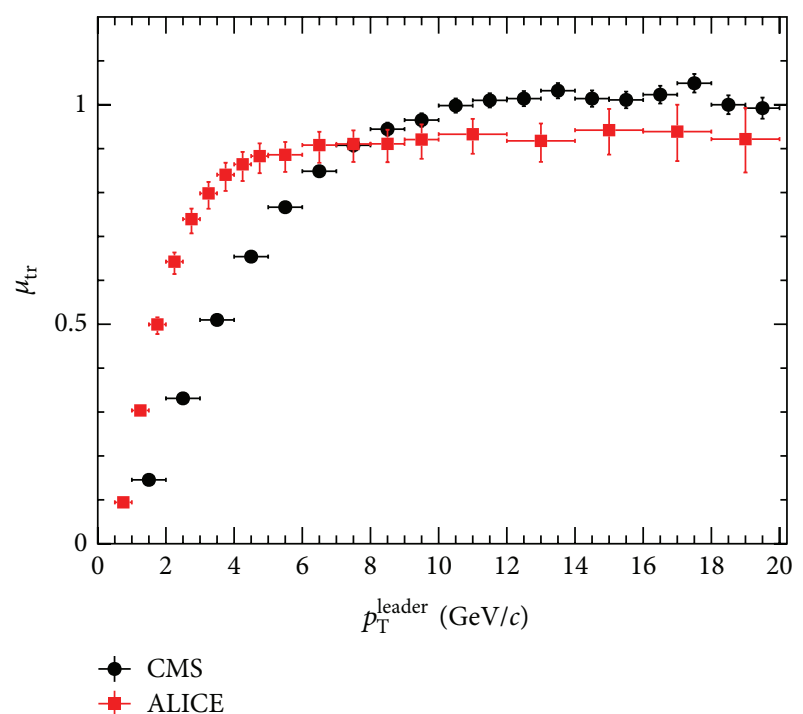

FIGURE 2: Charged-particle density in the transverse region as a function of $p_{T}$ of leading object [8] (CMS: charged particle jet, ALICE: charged particle). CMS analyses particles with $p_{T}>$ $0.5 \mathrm{GeV} / c$ and $|\eta|<2.4$; ALICE with $p_{T}>0.5 \mathrm{GeV} / c$ and $|\eta|<0.8$.

the charged particle density in the transverse region $60^{\circ}<$ $|\Delta \phi|<120^{\circ}$ defined as follows:

$$
\mu_{\mathrm{tr}}=\frac{N_{\mathrm{ch}}^{\mathrm{tr}}}{\Delta \eta \Delta(\Delta \phi)}
$$

where $N_{\mathrm{ch}}^{\mathrm{tr}}$ is the charged particle multiplicity in the transverse region, $\Delta \eta$ is the pseudorapidity range studied, and $\Delta(\Delta \phi)$ is the azimuthal width of the transverse region. This is really the case as shown in Figure 2.

Let us explain it. Starting from large transverse momenta of triggers on the right-hand side of Figure 2 and going to the left, we somehow feel at the beginning the central region from $b=0$ to the end of the plateau. Then the density of accompanying particles in the transverse region should not change until we approach the end of it. The difference in positions of the decrease in the two plots is defined by the difference in the choice of the leading trigger for two collaborations. Surely, this is a qualitative explanation. Further, more qualitative estimates should be done when higher precision data become available. The flat dependence of $\mu_{\mathrm{tr}}$ on $p_{T}$ shows that activity in the transverse region is independent of hard process scale, provided that scale is hard enough that all proton-proton interactions are central.

Some other characteristics of such inelastic processes considered in [8] support this conclusion. Usage of very high multiplicity events in combination with jet properties is crucial. In particular, significant reduction of jet rate at very high multiplicities compared to MC predictions asks for new inputs in the models. The simple increase of the geometrical overlap area of the colliding protons does not account for properties of jet production at very high multiplicities. It looks as if the parton (gluon) density must strongly increase in central collisions and rare configurations (fluctuations) of the partonic structure of protons are involved. The correlation studies of jets (see, e.g., [10]) can be used for further femtoscopy of the fine structured system.

The positivity of $G(s, b)$, that is, of $\sigma_{\text {inel }}(s, b)$, imposes some limits on the relative role of $B$ and $\sigma_{t}$. It follows from (15) that

$$
2 Z=\frac{8 \pi B}{\sigma_{t}}=\frac{\sigma_{t}}{2 \sigma_{\mathrm{el}}} \geq 1 ;
$$

that is, the slope $B$ should increase asymptotically at least as strong as the total cross section $\sigma_{t}$.

This inequality is fulfilled at present and intermediate energies. If the value of $Z$ will decrease at energies above $7 \mathrm{TeV}$, as one could expect from its tendency shown in Table 1 , and approach $Z=0.5$ this inequality can be saturated. We discuss first what happens in the region $0.5 \leq Z \leq 1$. The values $Z<0.5$ will be discussed at the end.

It is usually stated that the equality $2 Z=8 \pi B / \sigma_{t}=1$ corresponds to the black disk limit with equal elastic and inelastic cross sections $\sigma_{\mathrm{el}}=\sigma_{\mathrm{in}}=0.5 \sigma_{t}$. However, one sees from (15) that $G(s, b=0)=0$ at $Z=0.5$; that is, no inelastic interactions take place in central collisions. Surely, one should not call it as a black disk. This paradox is resolved [11] if we write the inelastic profile of the interaction region using (14). At $Z=0.5$ it looks like

$$
G(s, b)=4\left[\exp \left(-\frac{b^{2}}{2 B}\right)-\exp \left(-\frac{b^{2}}{B}\right)\right] .
$$

We see that one must rename the black disk as a black toroid (or a black ring in the two-dimensional projection) with full absorption $G\left(s, b_{m}\right)=1$ at the impact parameter $b_{m}=\sqrt{2 B \ln 2}$, complete transparency at $b=0$, and rather large half-width. Thus, the evolution to values of $Z$ smaller than 1 at higher energies (this can happen if the decreasing tendency of $Z$ with energy from ISR to LHC persists) would imply quite special transition from the two-scale features at the LHC to the concave torus-like configurations of the interaction region if the exponential shape of the diffraction cone, described by (2), persists.

It looks as if the protons penetrate through one another at central collisions, just scattering elastically, while peripheral collisions become responsible for inelastic processes. They begin to prevail over elastic scattering only at the periphery $b>b_{m}$. Is the parton coherence inside each colliding proton responsible for that?

A signature of transition to such regime at $7 \mathrm{TeV}$ at the level $10^{-4}$ in a single point was claimed in [12] while the accuracy of measuring $Z$ is about $10^{-3}$. Some depletion of $G$ was demonstrated in a plot of [12] shown in Figure 3.

No such depletion was observed in [5] where the values of $G(b)$ decrease steadily from 1 at $b=0$. Different results of $[5,12]$ using the fit by the same model $[6]$ prevent from any (even preliminary) statements. The value of $Z$ is equal to 1 at $7 \mathrm{TeV}$ within the experimental inaccuracy of measuring the slope $B$ and the total cross section $\sigma_{t}$. This inaccuracy could be the reason for the disagreement in conclusions of $[5,12]$. The depletion is seen in Figure 3 at the fourth digit only what is surely inside the scope of experimental error bars. 


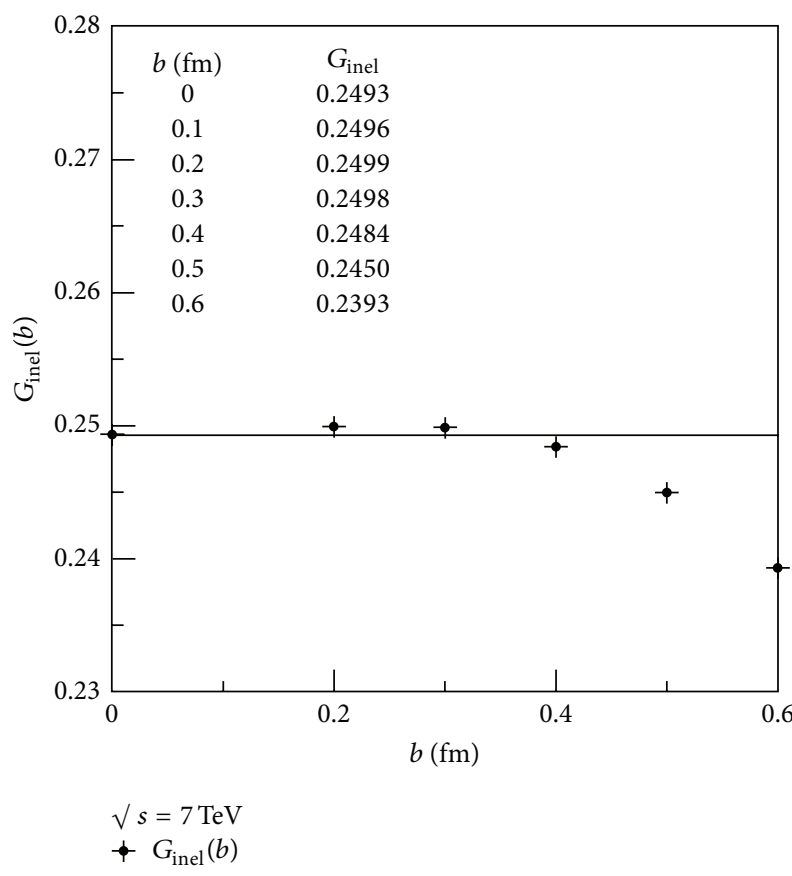

FIgURE 3: The impact parameter dependence of the function $G_{\text {inel }}(b)=0.25 G(b)$ at $7 \mathrm{TeV}$ [12]. It is obtained using the fit of experimental data according to the model [6].

Nevertheless, the further energy evolution of $Z$ should be carefully followed. Its implications for inelastic processes are of great interest. The mean multiplicity will, probably, decrease because of the more peripheral origin of newly created particles. Also, jets will become produced at the periphery in distinction to the situation described above. That would imply that they will have to penetrate larger distances in transverse direction compared to forward directions. It would give rise to their stronger depletion in the transverse plane and, therefore, to the azimuthal asymmetries which were looked for in [10]. Surely, there will be found other criteria of transition to the concave shape of the interaction region of inelastic processes.

One cannot exclude another possibility when, for example, the black region will extend to larger impact parameters at higher energies. However, this should result immediately in the drastic change of the shape of the diffraction cone declining strongly from the simple exponential parameterization exploited above. Maybe, it would be even more exotic behavior. One could speculate on its relation to the increase of the total cross section with energy.

In principle, the positivity of the inelastic cross section

$$
\sigma_{\text {in }}=\frac{\pi B}{Z^{2}}(4 Z-1) \geq 0
$$

admits the value of $Z$ as small as 0.25 which corresponds to $\sigma_{\text {el }}=\sigma_{t}$ and $\sigma_{\text {in }}=0$. The values of $Z<0.5$ lead to negative values of $\sigma_{\text {in }}(s, b)$, that is, to negative FourierBessel transforms of $g(p, \theta)$ in (5). They are not forbidden if the relative phases of matrix elements of inelastic processes $M_{i}$ in (5) interfere in such a way. Unfortunately, we have no knowledge about them. This possibility was treated as another branch of the solution of the unitarity condition and named as antishadowing or refractive scattering in [13] and as resonant disk modes in [14]. However, this regime is, surely, shifted to extremely high energies if it can be observed at all. The approach to asymptotics is argued as following the logarithmic dependences of cross sections $\sigma_{t} \propto \sigma_{\text {el }} \propto \ln ^{2} s$ and $\sigma_{\text {in }} \propto \ln s$. The depletion of $G(s, 0)$ in [12] was ascribed to this regime by mistake while the values of $Z$ are near 1 at $7 \mathrm{TeV}$.

At the very end, let me briefly mention the problem of the ratio of real and imaginary parts outside the diffraction cone. The unitarity condition is quite successful in this interval of angles as well. It was shown long ago $[1,15]$ that the imaginary part of the amplitude $f$ outside the diffraction cone can be derived from the general unitarity condition (3) which is reduced there to the inhomogeneous linear integral equation:

$$
\begin{aligned}
\operatorname{Im} & f(p, \theta) \\
= & \frac{p \sigma_{t}}{4 \pi \sqrt{2 \pi B}} \\
& \cdot \int_{-\infty}^{+\infty} d \theta_{1} \exp \left(-\frac{B p^{2}\left(\theta-\theta_{1}\right)^{2}}{2}\right) r_{\rho} \operatorname{Im} f\left(p, \theta_{1}\right) \\
& +g(p, \theta),
\end{aligned}
$$

where $r_{\rho}=1+\rho(s, 0) \rho\left(s, \theta_{1}\right)$. This reduction becomes possible because the contribution from asymmetrical configuration of scattering angles in the first term of (3) dominates due to the steep Gaussian falloff inside the diffraction cone. Because of the sharp falloff of the amplitude with angle, the principal contribution to the integral arises from a narrow region around the line $\theta_{1}+\theta_{2} \approx \theta$. Therefore one of the amplitudes should be inserted at small angles within the cone as a Gaussian while another one is kept at angles outside it.

It can be solved analytically (for more details see $[1,15]$ ) with two assumptions that the role of the overlap function $g(p, \theta)$ is negligible outside the diffraction cone and the function $r_{\rho}$ may be approximated by a constant; that is, $\rho\left(\theta_{1}\right)=\rho_{l}=$ const. Its solution prescribes the exponential (the exponent is predicted in the analytical form!) decrease with $\sqrt{|t|}$ plus imposed on it damped oscillations responsible for the dip of the differential cross section:

$$
\begin{aligned}
\operatorname{Im} & f(p, \theta) \\
= & C_{0} \exp \left(-\sqrt{2 B \ln \frac{Z}{r_{\rho}}} p \theta\right) \\
& +\sum_{n=1}^{\infty} C_{n} \exp \left(-\left(\operatorname{Re} b_{n}\right) p \theta\right) \cos \left(\left|\operatorname{Im} b_{n}\right| p \theta-\phi_{n}\right)
\end{aligned}
$$

with

$$
b_{n} \approx \sqrt{2 \pi B|n|}(1+i \operatorname{sign} n) \quad n= \pm 1, \pm 2, \ldots
$$

The only unknown ingredient in the predicted exponent is the ratio of real to imaginary parts of the amplitude outside 
the diffraction cone $\rho_{l}$. Replacing it by its average value there one is able to fit experimental data. The great surprise of the fit of TOTEM data at $7 \mathrm{TeV}$ [16] was a necessity to use there the large (in modulus) negative value of this ratio $\rho_{l} \approx-2.1$ (if $\rho_{0}=0.14$ ) not predicted by phenomenology that poses another problem. It is required by the increased (compared to lower energies) slope in this region.

No zeros of the imaginary part appear in this approach while all phenomenological models ask for such a zero there. This problem has not been resolved up to now.

\section{Conflict of Interests}

The author declares that there is no conflict of interests regarding the publication of this paper.

\section{Acknowledgments}

The author is grateful for support by the RFBR Grants 12-02-91504-CERN-a and 14-02-00099 and the RAS-CERN program.

\section{References}

[1] I. V. Andreev and I. M. Dremin, "Large-angle elastic scattering," Pis'ma v ZhETF, vol. 6, p. 810, 1967.

[2] I. M. Dremin, "Elastic scatteringof hadrons," Uspekhi Fizicheskikh Nauk, vol. 183, no. 1, pp. 3-28, 2013.

[3] I. M. Dremin, "Elastic scattering of hadrons," Physics-Uspekhi, vol. 56, no. 1, pp. 3-28, 2013.

[4] L. van Hove, "A phenomenological discussion of inelastic collisions at high energies," Il Nuovo Cimento, vol. 28, no. 4, pp. 798-817, 1963.

[5] I. M. Dremin and V. A. Nechitailo, "Proton periphery activated by multiparticle dynamics," Nuclear Physics A, vol. 916, pp. 241248, 2013.

[6] U. Amaldi and K. R. Schubert, "Impact parameter interpretation of proton-proton scattering from a critical review of all ISR data," Nuclear Physics B, vol. 166, no. 2, pp. 301-320, 1980.

[7] L. Frankfurt, M. Strikman, and C. Weiss, "Transverse nucleon structure and diagnostics of hard parton-parton processes at LHC," Physical Review D, vol. 83, Article ID 054012, 2004.

[8] M. Y. Azarkin, I. M. Dremin, and M. Strikman, "Jets in multiparticle production in and beyond geometry of protonproton collisions at the LHC," Physics Letters B, vol. 735, pp. 244-249, 2014.

[9] S. Chatrchyan, V. Khachatryan, A. M. Sirunyan et al., "Jet and underlying event properties as a function of charged-particle multiplicity in proton-proton collisions at $\sqrt{s}=7$," The European Physical Journal C, vol. 73, article 2674, 2013.

[10] The CMS Collaboration, preprint PAS FSQ-13-005, 2013.

[11] I. M. Dremin, "Critical regime of proton elastic scattering at the LHC, JETP Letters, vol. 99, no. 5, pp. 243-245, 2014.

[12] A. Alkin, E. Martynov, O. Kovalenko, and S. M. Troshin, "Impact-parameter analysis of TOTEM data at the LHC: black disk limit exceeded," Physical Review D, vol. 89, Article ID 091501(R), 2014.

[13] S. M. Troshin and N. E. Tyurin, "Beyond the black disk limit," Physics Letters B, vol. 316, no. 1, pp. 175-177, 1993.
[14] V. V. Anisovich, V. A. Nikonov, and J. Nyiri, "Hadron collisions at ultrahigh energies: black disk or resonant disk modes?" Physical Review D, vol. 90, Article ID 074005, 2014.

[15] I. V. Andreev and I. M. Dremin, "Large-angle scattering at high energies," Soviet Journal of Nuclear Physics, vol. 8, p. 473, 1969.

[16] I. M. Dremin and V. A. Nechitailo, "Elastic $p p$-scattering at $\sqrt{s}=7 \mathrm{TeV}$ with the genuine Orear regime and the dip," Physical Review D, vol. 85, Article ID 074009, 2012. 

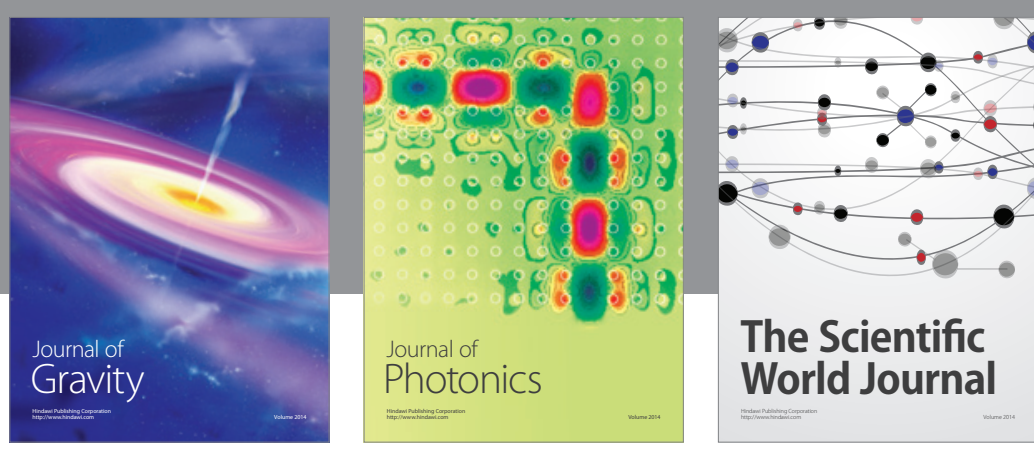

The Scientific World Journal
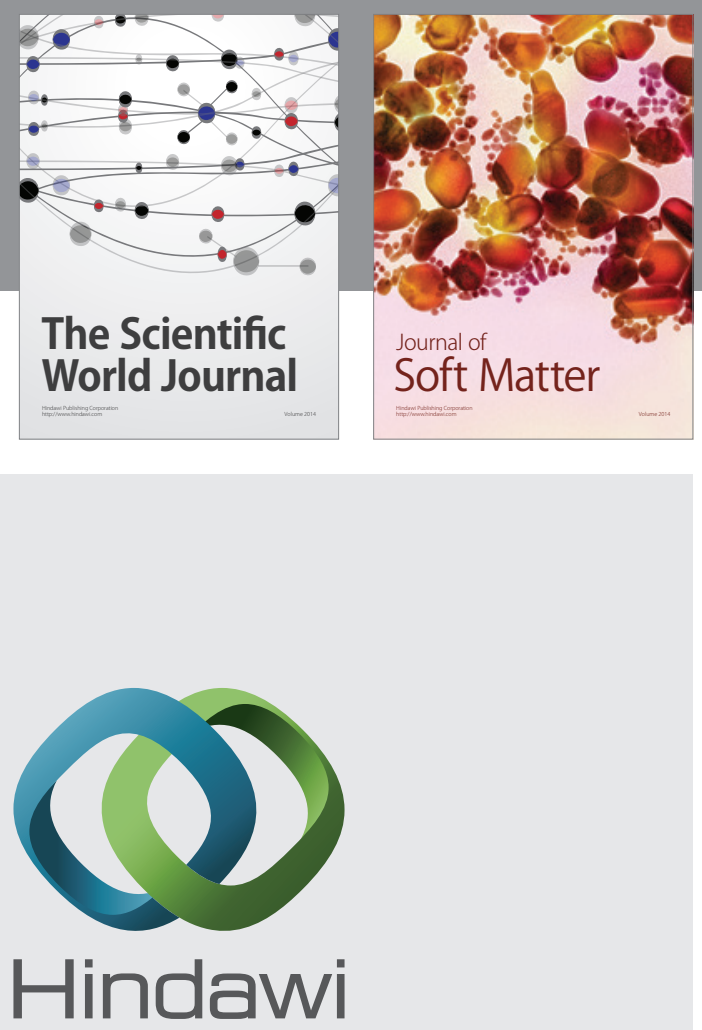

Submit your manuscripts at

http://www.hindawi.com

nternational Journal of

Statistical Mechanics
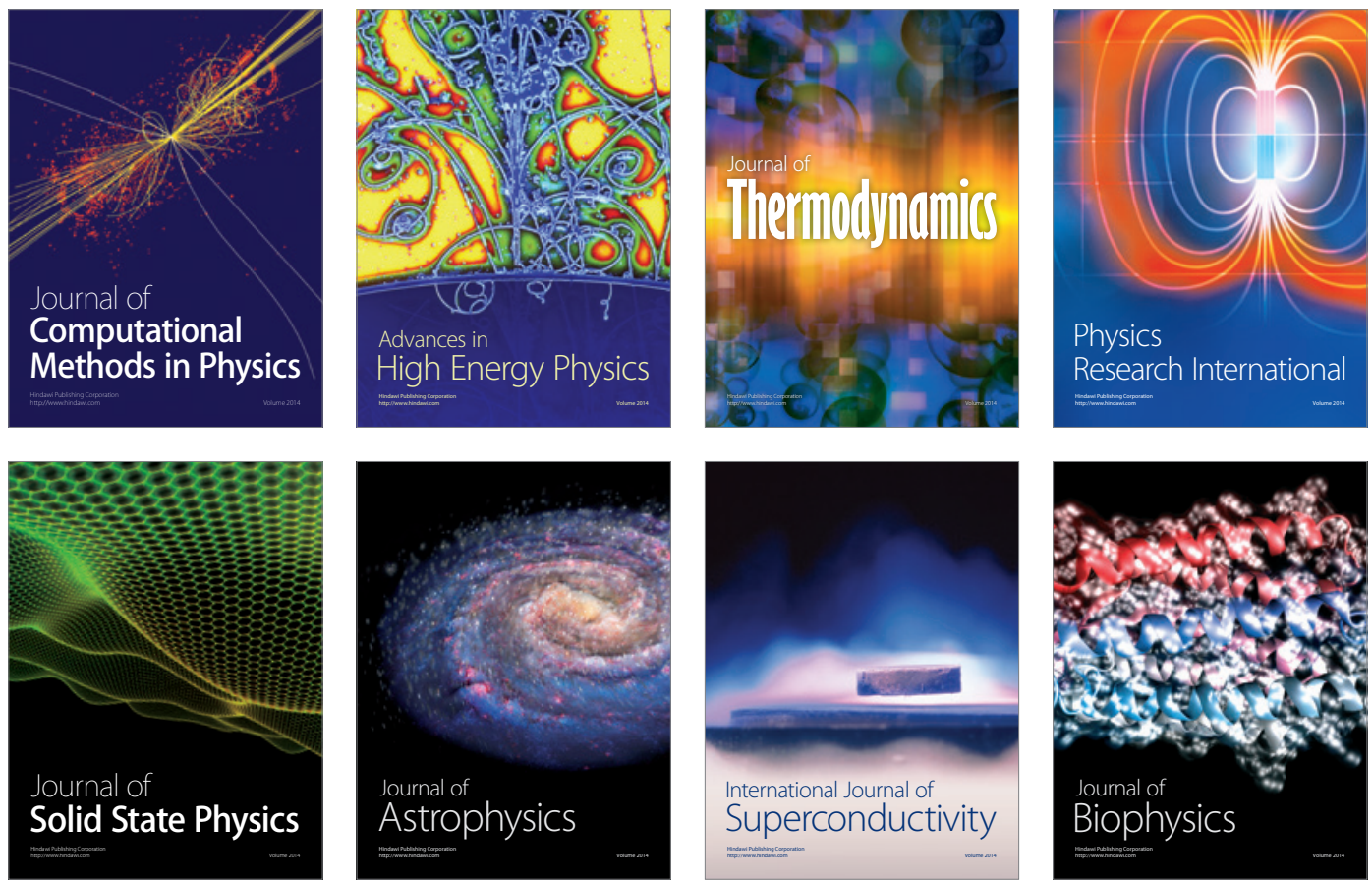
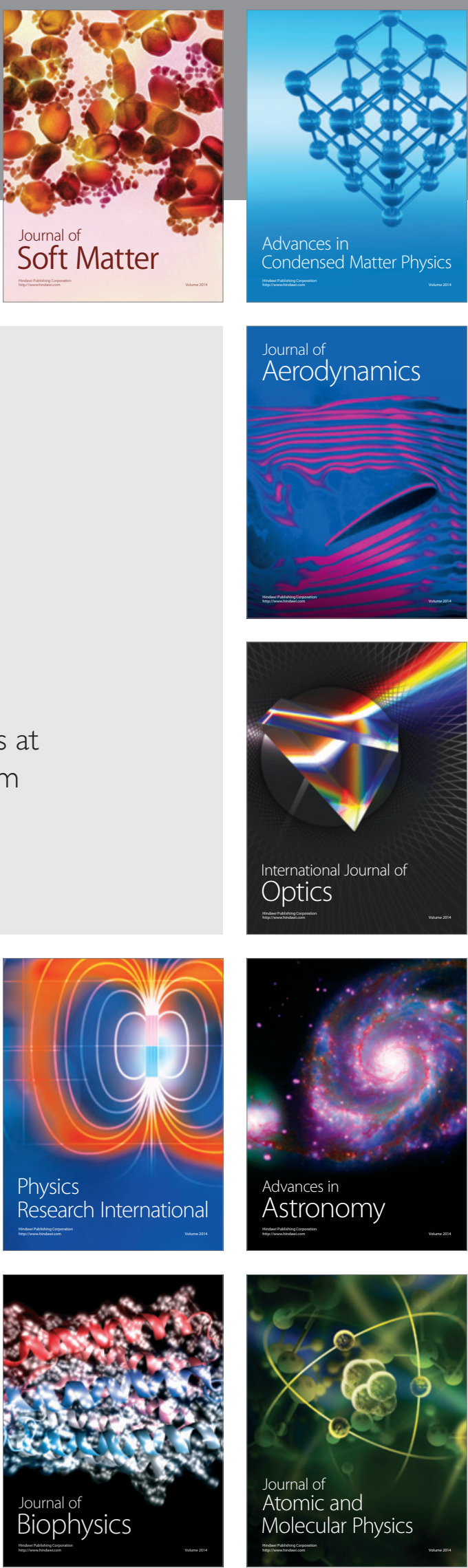